\title{
Short-term results of early switch from Ranibizumab to Aflibercept in poor or non responder age related macular degeneration in clinical practice
}

\author{
Luciana de Sá Quirino-Makarczyk¹, Maria de Fátima Sainz Ugarte ${ }^{2 *}$, Bruna Viana Vieira² ${ }^{2}$ Sérgio Kniggendorf ${ }^{1}$ \\ and Caio Vinicius Saito Regatieri ${ }^{3}$
}

\begin{abstract}
Background: To evaluate the change in best corrected visual acuity (VA) and central macular thickness (CMT) following treatment with intravitreal aflibercept (AFL) in patients poorly responders or non responders to ranibizumab (RBZ).

Methods: Charts of patients injected with RBZ from January 2016 to December 2018 (548 cases) due to neovascular age-related macular degeneration (nAMD) were reviewed. Fifty-six cases met our criteria for poor responders to RBZ (CMT decreased between 5 and 15\% over treatment) or for non responders to RBZ (CMT decreased less than 5\% or increased over treatment).

Results: After the third AFL injection, CMT decreased from $384.38 \pm 123.20 \mu \mathrm{m}$ to $296.18 \pm 70.52 \mu \mathrm{m}$ in the nonresponder group and from $320.00 \pm 82.05 \mu \mathrm{m}$ to $282.27 \pm 56.86 \mu \mathrm{m}$ in the poor responder group. Although decrease in macular thickness was overall achieved 3 months after switching to AFL, it was not translated in VA improvement.

Conclusions: it was observed that nAMD patients classified as RBZ non-responders tend to respond better to AFL than RBZ poor-responders anatomically, without correspondent improvement in VA.
\end{abstract}

Keywords: Aflibercept, Ranibizumab, Age-related macular degeneration, Vascular endothelial growth factor

\section{Introduction}

Age-related macular degeneration (AMD) is a degenerative pathology that can cause severe and permanent vision loss. The neovascular AMD is characterized by growth of abnormal vessels that lead to leakage of sub and intraretinal fluid. The growth of those abnormal vessels is mainly caused by an increase in the production of angiogenic factors, being the vascular endothelial growth factors (VEGF) the most important. VEGF activates angiogenesis and vascular permeability $[1,2]$.

*Correspondence: sainzfatima@gmail.com

${ }^{2}$ Hospital Oftalmológico de Brasília, Brasília, Brazil

Full list of author information is available at the end of the article
Vascular endothelial growth factors inhibitors have been used for neovascular AMD treatment. The most commonly used anti-VEGF factors in the clinical practice nowadays are: ranibizumab (Lucentis ${ }^{\circledR}$, Novartis), aflibercept (Eylea ${ }^{\circledR}$, Bayer) and bevacizumab (Avastin ${ }^{\circledR}$, Roche Pharma), although the use of the last one is off-label [2, $3]$.

Ranibizumab (RBZ) is a recombinant humanized IgG1 monoclonal antibody fragment $(48 \mathrm{kDa})$, that neutralizes all active forms of VEGF-A. Aflibercept (AFL) is a fusion protein $(115 \mathrm{kDa})$ that neutralizes all forms of VEGF-A, VEGF-B and placental growth factor 1 and 2 . In vitro studies showed that AFL has a longer half-life than ranibizumab [4]. Regarding to the affinity of these drugs to VEGF, studies showed some conflicting results [5]. 
The safety and efficacy of RBZ as intravitreal injection for neovascular AMD (nAMD) was shown in several multicentric studies $[6,7]$, as monthly or pro re nata protocols (PRN) [8]. Although some patients achieved positive results with the treatment, several others continued to experience progressive visual loss and macular fluid even with monthly intravitreal injections. The first and second years of CATT study showed that more than $50 \%$ of patients that received RBZ had macular fluid persistence after the twelfth month period of analysis $[9,10]$. Those patients can be defined as resistant to treatment. Although the resistance mechanisms are not explained yet, tolerance and tachyphylaxis to RBZ are the possible causes [11]. The HARBOR study showed that higher doses of RBZ were not superior if compared to the $0.5 \mathrm{mg}$ dose and that monthly administration leads to superior results than PRN protocol [12]. Contradicting HARBOR study, SAVE study showed that loss of efficacy can be resolved, in some patients, using higher doses [13].

The studies VIEW 1 and VIEW 2 compared the efficacy and safety of monthly intravitreal injections of RBZ and AFL and concluded that they were equally efficient to improve and prevent vision loss. Although the group treated with AFL needed an average of five less injections after 96 weeks [14].

Recently, several studies discussed the development of anti-VEGF resistance and have demonstrated that the switch of intravitreal drug can be effective in treatment [15-18].

Our goal in this study was to compare visual and macular results over the first 3 months of treatment after early switch to aflibercept in the treatment of neovascular AMD with complete or partial resistance to RBZ.

Our secondary goal was to analyze if the poor responders and non responders groups differ in tomographic macular findings when injected with AFL.

\section{Methods}

This study was approved by Research Ethics Committee of Hospital Oftalmológico de Brasília and it was performed in accordance to the Declaration of Helsinki that sets out rules on research involving humans.

We retrospectively evaluated patients with nAMD that were injected with RBZ and posteriorly treated with AFL between January 2016 to December 2018. Patients identified as poor or non-responders to RBZ treatment were selected for more detailed analysis.

All charts selected for analysis were from patients who received $0.5 \mathrm{mg}$ of intravitreal RBZ for 3 months before early switch to AFL. Also, after drug switch, all selected patients had to meet the criteria of at least 3 monthly loading doses of AFL $2 \mathrm{mg}$.
Patients were imaged using the Heidelberg Spectralis (Heidelberg Engineering, Germany ${ }^{\circledR}$ ) in spectral domainoptical coherence tomography (OCT) mode. Automatic quantitative maps of retinal thickness were evaluated: a baseline map and a map 1 month after each injection.

Patients included were considered, according to OCT findings, either: (1) poor-responsive, whose central macular thickness minimally changed (5-15\%) over treatment with RBZ or (2) non-responsive, whose central macular thickness did not change $(<5 \%)$ or increased over treatment with RBZ.

The included patients should be pseudophakic, with visual acuity better than $1.3 \operatorname{logMAR}$ (visual acuity scale). In cases with bilateral pathology, both eyes could be included in the study if they met the inclusion criteria. The interval between the last RBZ injection and the first AFL injection should be superior to 4 and inferior to 6 weeks.

The exclusion criteria were: patients with macular epiretinal membranes, glaucoma, neovascular subretinal membranes from another etiology, foveal fibrosis or retinal pigment epithelium (RPE) tears. In none of the patients included in this study RPE detachments were a parameter to start treatment.

Collected data from patients included in this study were age, gender, best corrected vision acuity (VA), OCT morphology and central macular thickness (CMT), complications and adverse events associated. VA was measured with Snellen chart, and the decimal visual acuity was converted to the logarithm of the minimal angle of resolution $(\log M A R)$ units for the statistical analysis. Baseline mean VA and CMT were compared with their outcomes one month after each injection. Comparisons of mean VA and CMT changes between treatments after ranibizumab and aflibercept injections were done. Data are presented as mean and standard deviations.

Distribution of data for normality was checked using Shapiro-Wilk test. The significance of any difference in means was evaluated by nonparametric test (MannWhitney) and for parametric analysis the T-test was used. Statistical significance was defined as $\mathrm{p}<0.05$.

\section{Results}

Five hundred forty-eight charts of patients treated with intravitreal injections during January 2016 to December 2018 were reviewed. Fifty-six patients met the inclusion criteria. Among those, 11 were considered poor responders and 45 were considered non responders to RBZ treatment.

The average age was 70.3 years old (ranged from 53 to 93 years old). Sixty-six per cent $(66.07 \%)$ were female and 


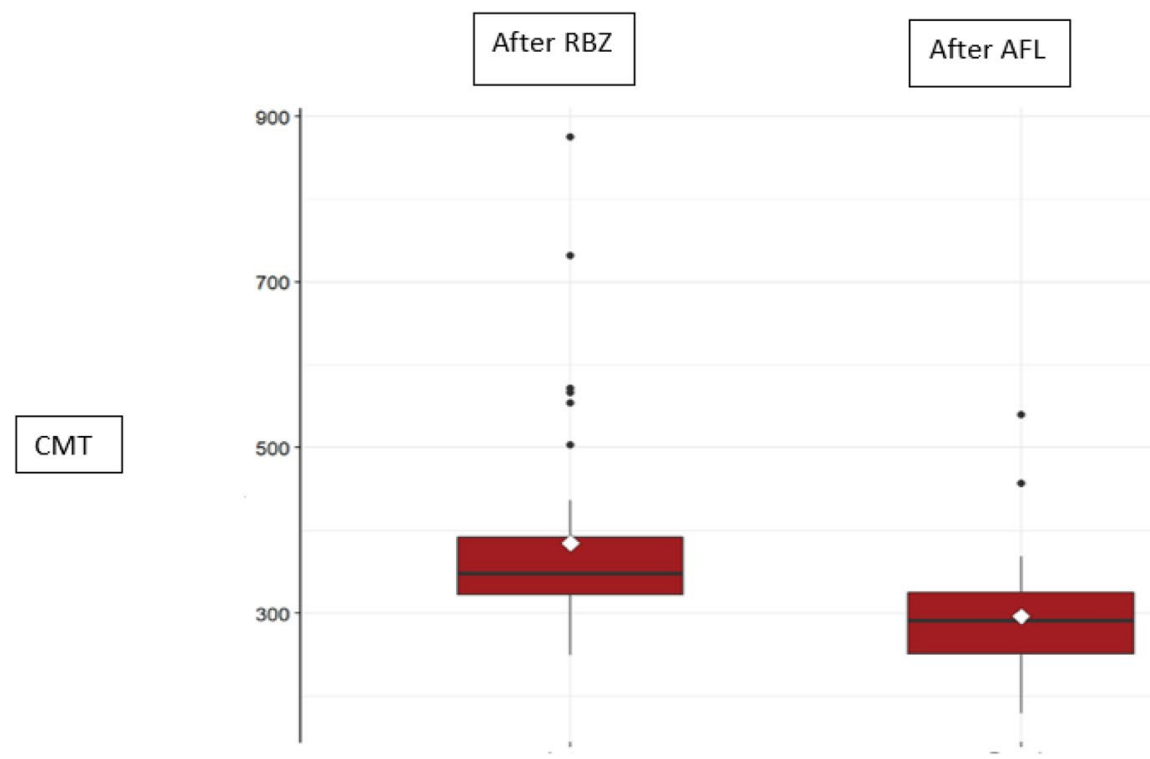

Fig. 1 Central macular thickness (CMT) of the ranibizumab (RBZ) non-responder patients after the 3rd injection with RBZ (mean: $384.38 \pm 123.20 \mu \mathrm{m}$ ) and after the 3rd injection with aflibercept (AFL) (mean: 296.18 $\pm 70.52 \mu \mathrm{m}$ )

thirty-three per cent (33.09\%) were male. Before switching to AFL, the VA ranged from 0.1 to $1.3 \log M A R$.

Figure 1 shows box plots comparing mean central macular thickness of the ranibizumab non-responder patients after the third injection of ranibizumab and then after the third injection of aflibercept.

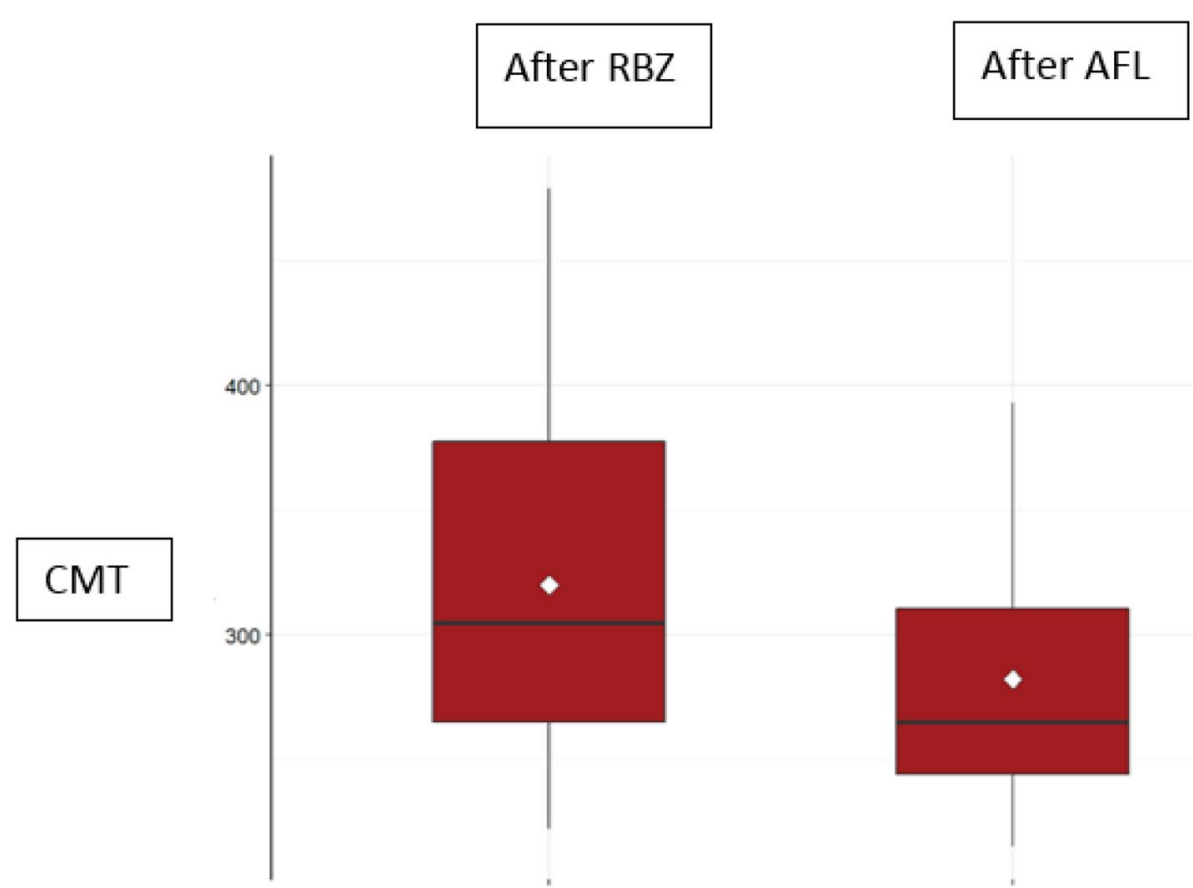

Fig. 2 Central macular thickness (CMT) of the ranibizumab (RBZ) poor-responder patients after the 3rd injection with RBZ (mean: $320.00 \pm 82.05 \mu \mathrm{m}$ ) and after the 3rd injection with aflibercept (AFL) (mean: $282.27 \pm 56.86 \mu \mathrm{m}$ ) 
Figure 2 shows box plot comparing mean CMT of the RBZ poor-responder group after the third injection of RBZ and then after the third injection of AFL.

The Mann-Whitney test was used to compare final CMT after the two treatments. In both groups, the CMT decreased significantly after last injection of AFL as compared to the values measured 1 month after the last RBZ injection $(\mathrm{p} \leq 0.05)$. However, this difference was more significant in the group of non-responders (p-value $<0.01)$ than in the group of poor responders $(\mathrm{p}$-value $=0.01247)$.

Analysis of the CMT monthly variation (baseline and 1 month after each injection) using Wilcoxon test showed that the results obtained are mainly due to the first injection of AFL in both groups ( $\mathrm{p} \leq 0.05)$. The CMT monthly variation, Fig. 3, confirmed that the non responder group had a better response than the poor responder group and that this difference was evident after the first dose of AFL ( $p=0.00000045$ for the non responder group and $\mathrm{p}=0.0021$ for the poor responder group). The second and third injections of AFL did not induce additional statistically significant differences in CMT.

There was no improvement observed in VA (measured in $\log M A R$ ) after third injection of AFL when compared to the last VA after RBZ treatment neither in the nonresponder group (Fig. 4) nor in the poor responder group (Fig. 5).

The total of patients, poor or non-responders to RBZ, were divided after treatment with AFL in groups of poor responders, non responders and responders to AFL. In order to compare proportions between these patients, a two-way table of categorical variables was created (Table 1). According to this table, most of the RBZ nonresponder patients, responded to AFL (48.9\%). On the other hand, most of the RBZ poor-responder patients, did not respond to AFL (54.5\%).

From the baseline OCT, it was observed that all the patients had disorganization of the outer retinal layers, including ellipsoid layer disruption, except by one patient of the non responder group. Additionally, 9 of the 11 patients of the poor responder group and 39 of the 45 patients from the non responder group had large neovascular membranes ( $\geq 4$ disc areas). Based on OCT and fluorescein angiogram characteristics, in the group of non responders: $55.11 \%$ of membranes (23 eyes) were mixed type and $55.55 \%$ ( 24 eyes) were type 2 . While in the group of poor responders, $54.54 \%$ of membranes $(6$ eyes) were mixed type and $45.45 \%$ ( 5 eyes) were type 2 . No signs of chronicity such as outer retinal tubulations where found in any of the groups.

In all 548 patients injected from January 2016 to December 2018, no adverse effects or complications were observed, such as endophthalmitis, retinal detachments, uveitis or increase in intraocular pressure. Also, there were no systemic adverse events.

Figure 6 shows a series of OCT-Scan images of a patient who partially responded to RBZ and, after the switch to AFL, responded completely.

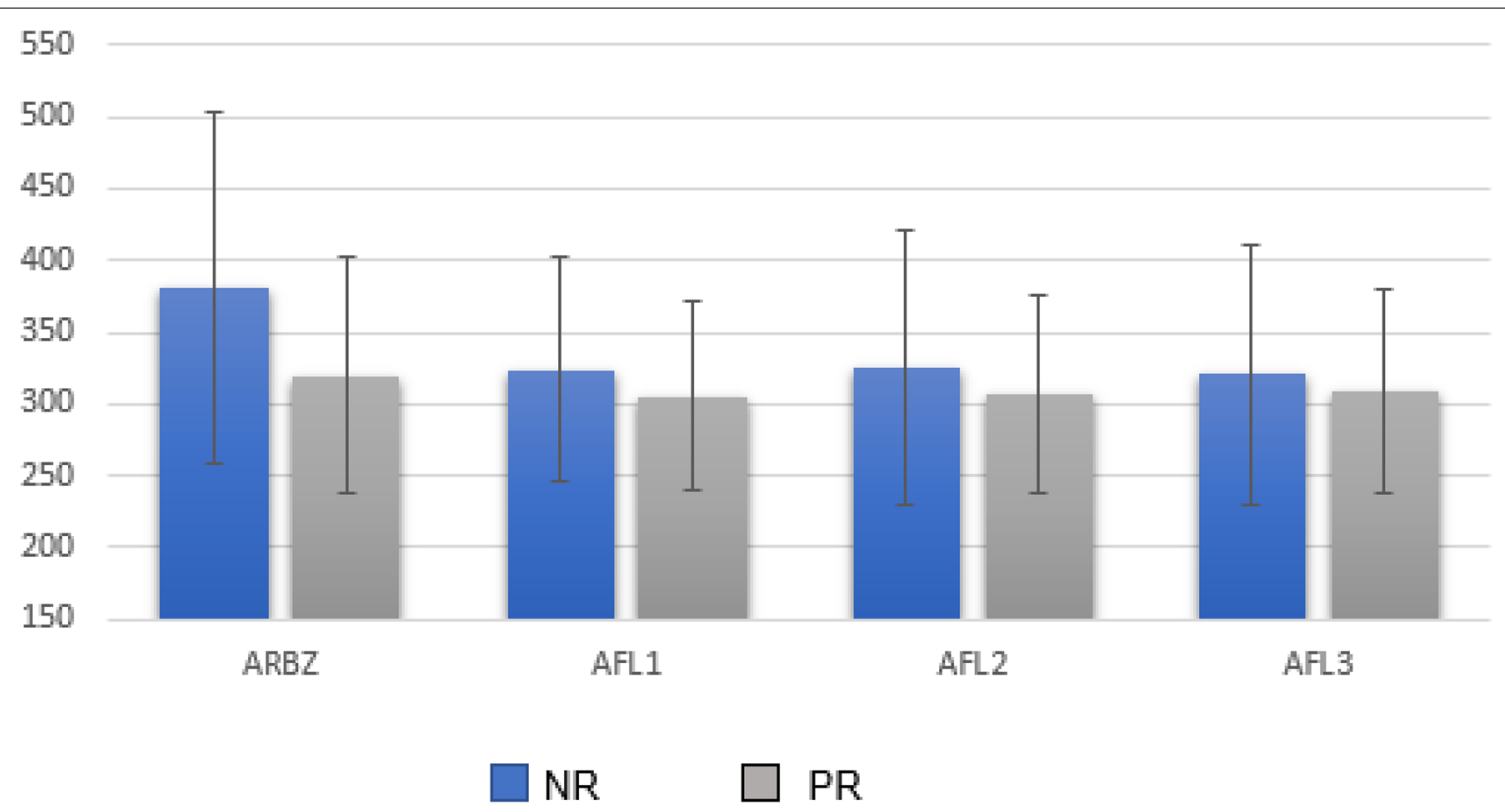

Fig. 3 Central macular thickness (CMT) monthly variation, measured in $\mu$ m, after the third dose of ranibizumab (ARBZ), after the first dose of Aflibercept (AFL1), after the second dose of aflibercept (AFL2), after the third dose of aflibercept (AFL3); NR non-responder, PR poor responder 


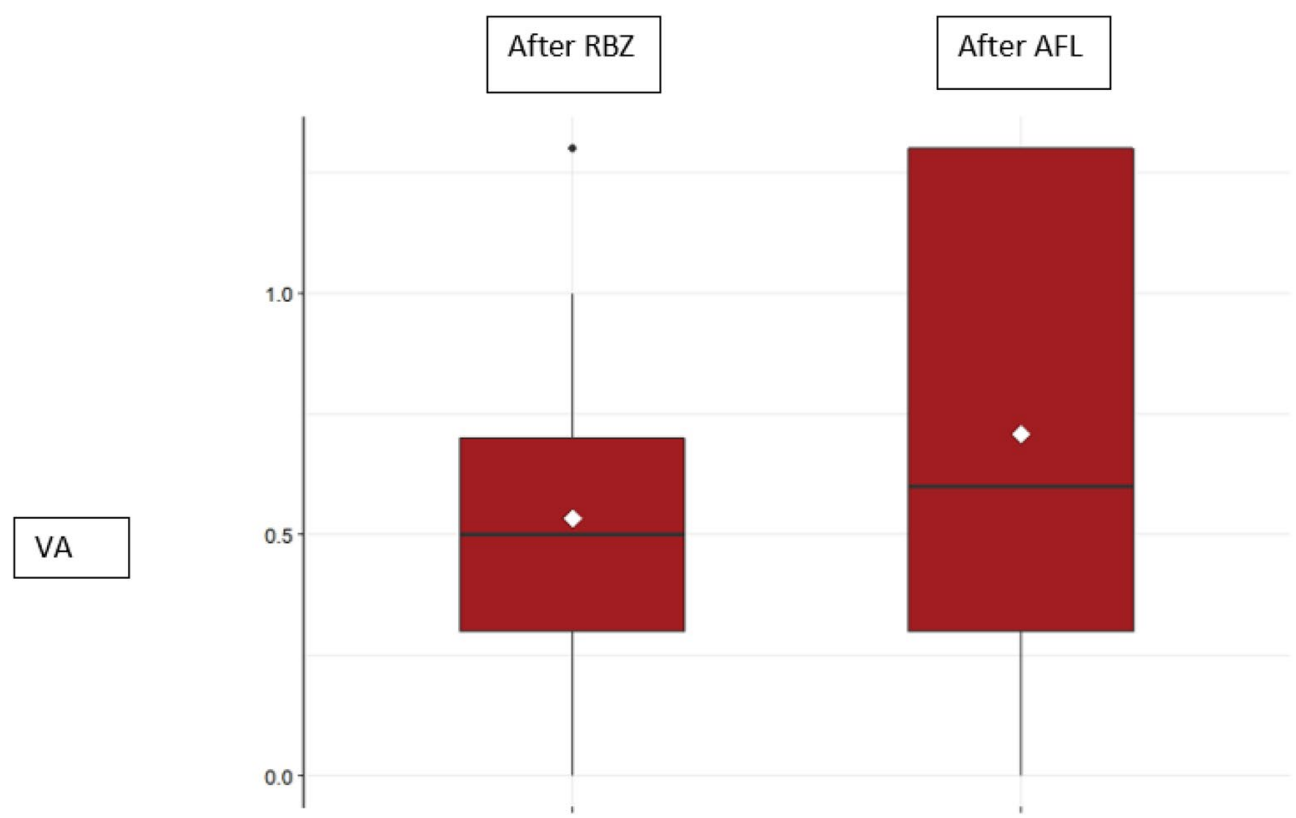

Fig. 4 Visual acuity (VA) of the ranibizumab (RBZ) non-responder patients after the 3rd injection of RBZ (mean: $0.53 \pm 0.36$ logMAR) and after the 3rd injection of aflibercept (AFL) (mean: $0.71 \pm 0.44 \log M A R$ )

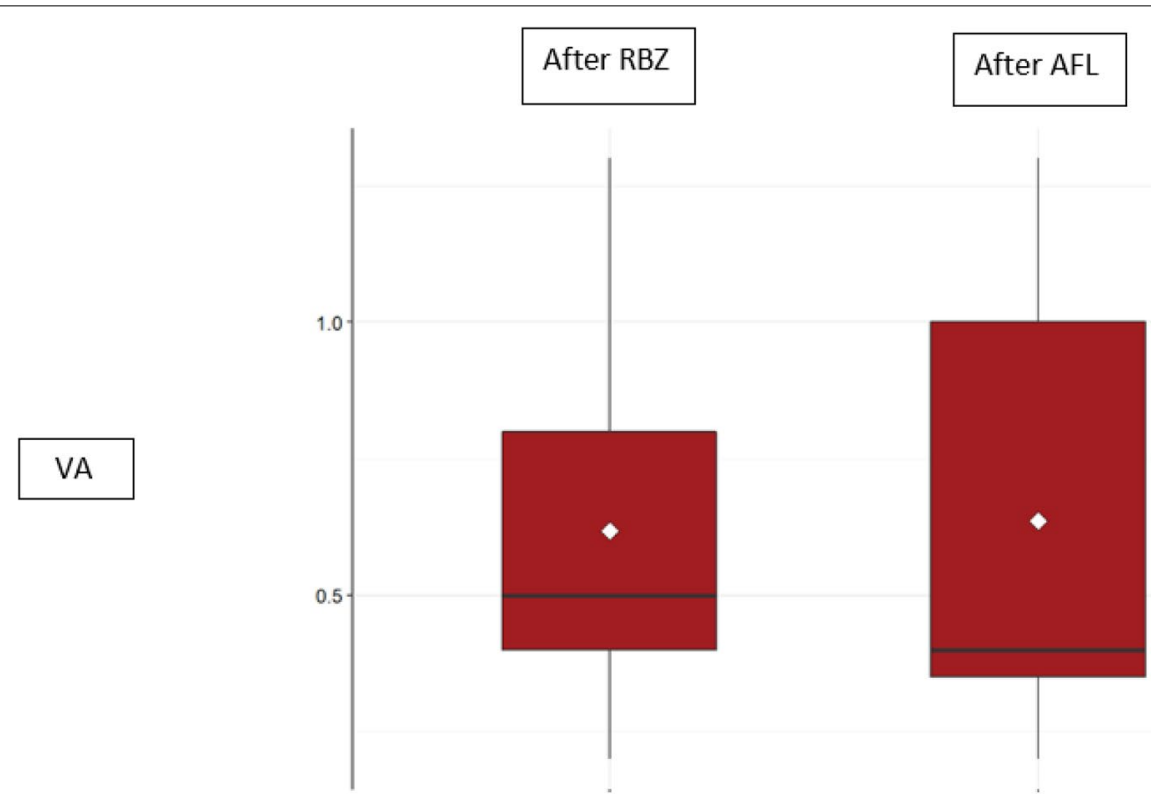

Fig. 5 Visual acuity (VA) of the ranibizumab (RBZ) poor responder patients after the 3rd injection of RBZ (mean: $0.62 \pm 0.33$ logMAR) and after the 3rd injection of aflibercept (AFL) (mean: $0.64 \pm 0.44 \log M A R$ )

\section{Discussion}

This study showed CMT improvement after treatment with intravitreal AFL in patients that were poor or non responders to RBZ, as found in other studies $[19,20]$. However, the group of non responder patients was proven to have a better response to AFL than the group of poor responder patients. Visual acuity did not improve neither in the non-responsive nor in the poor responsive group after the third injection of AFL when compared to the last visit after treatment with 3 doses of RBZ.

The decision of switching anti-VEGF agents occurred on the follow up after the third injection of RBZ, which 
Table 1 A two-way table is used to compare proportions of the final number of poor responders, non responders and responders after treatment with aflibercept (AFL) in the ranibizumab (RBZ) non responder and poor responder groups

\begin{tabular}{lcccc}
\hline & AFL non-responder group & AFL poor-responder group & AFL responder group & Total \\
\hline RBZ non-responder group & 9 & 14 & 22 & 45 \\
RBZ poor-responder group & 6 & 2 & 3 & 11 \\
Total & 15 & 16 & 25 & 56 \\
\hline
\end{tabular}

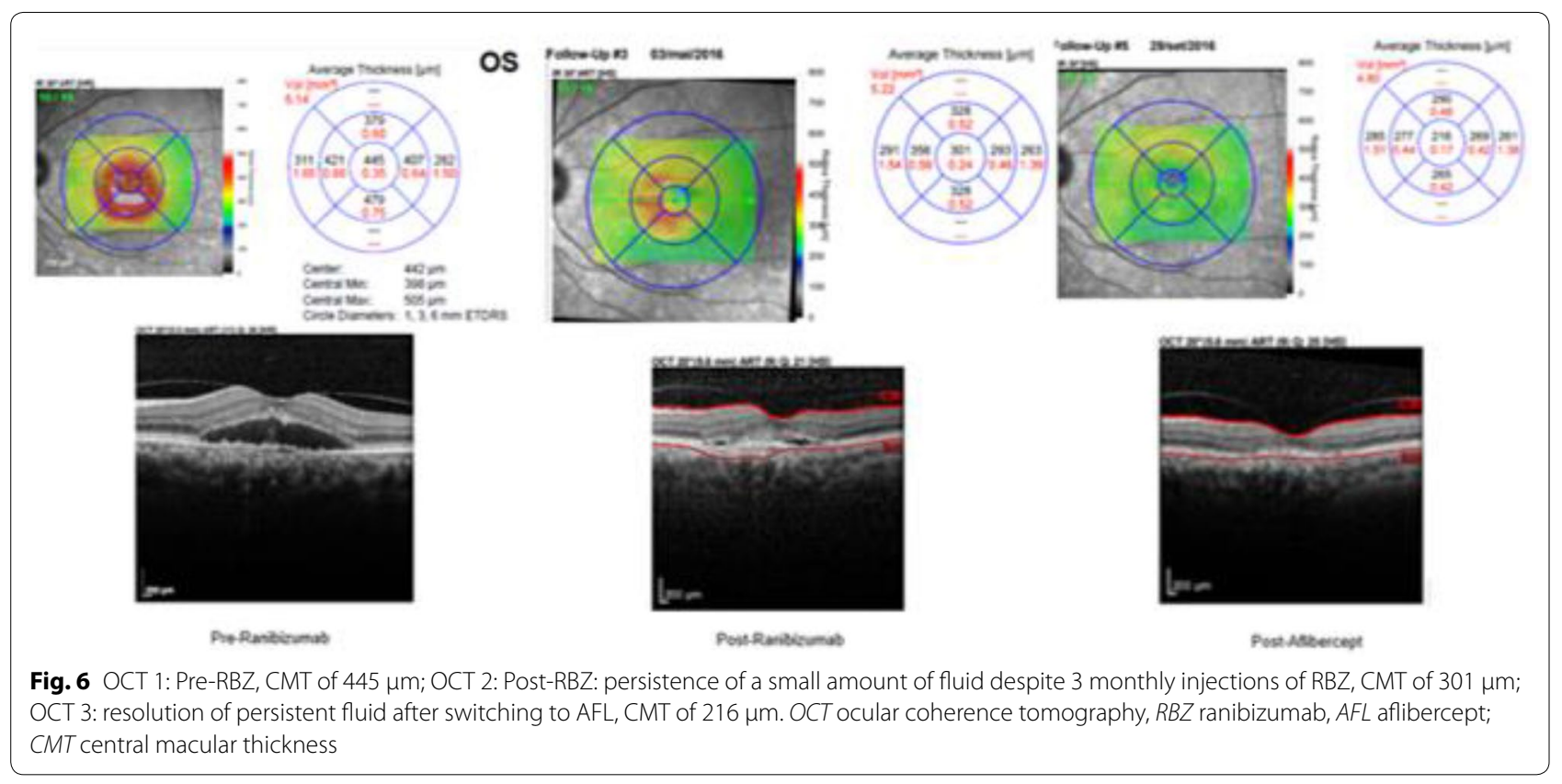

was proven not to be fully efficient. The early decision of switching anti-VEGF agents probably diminishes the visual harmful effects of the prolonged structural macular changes, allowing the patients to achieve their best potential vision. It was demonstrated that visual gain in the first 12 weeks treating macular edema was correlated to better final visual results, while the rapid improvement of CMT did not show to be predictive of better long term visual acuity [21]. Several studies did not demonstrate harmful effects to photoreceptors due to long term VEGF blockage [22, 23]. However, it has been hypothesized whether the extension of this effect could damage photoreceptors [24].

In this study, the majority of choroidal neovascular membranes were large and mixed type on baseline OCT. The membrane characteristics play a role on treatment outcome. Type 1 [19] and large [20, 21] choroidal neovascular membranes were defined as bad predictors for treatment outcome [19] as well as for poor response at 12 weeks $[20,21]$.
The results of this study could indicate a scenario of mainly two distinct responses to RBZ: (1) poor responders, where a possible tachyphylaxis or tolerance to RBZ had occurred and, due to a cross-tolerance effect, also a tendency to respond incompletely to AFL; (2) non responders, where VEGF-A is not the main causing factor for the neovascular growth.

Tachyphylaxis can occur after an initial dose or after series of drug doses [22-25]. Since tachyphylaxis cannot be overcome by increasing dosage, switching to a similar drug with different properties is recommended. Another reason for the poor response would be drug tolerance that could be caused by an over expression of VEGF receptors [11], which would result in reduced antiVEGF effects. In both circumstances, a partial response would happen with initial injections of RBZ, but the switch to AFL would improve the results. In the RBZ non responder group, an early switch to AFL would have been even more beneficial than in the poor responder group, rising the hypothesis that VEGF-B is more implicated in the pathophysiology. 
In almost all the studies regarding nAMD treatment, the presence of fluid on OCT was as a marker of disease activity and, therefore, indicated the need of retreatment. It was found that intraretinal fluid has a worse impact on VA if compared to subretinal fluid, especially when the fluid affects the fovea [24-27]. It was hypothesized that the refractory intraretinal fluid was caused by non-VEGF-mediated mechanisms, such as apoptotic or necrotic cell death [24], and was associated to a higher risk of fibrosis and geographic atrophy [25].

Controversially, the presence of subfoveal subretinal fluid may be associated with better visual outcomes and vision maintenance in the long term [26-29], since it would not represent neovascular activity, but a residual anatomical space caused by the failure of the neurosensory retina to reattach to the retinal pigment epithelium. One of the possible reasons for this is that the outer retinal layers in patients presenting with subfoveal subretinal fluid are less disrupted than in patients with intraretinal fluid involving the fovea [30]. Additionally, subretinal fluid could have a protective effect against geographic atrophy [31].

\section{Conclusions}

In conclusion, the early switch of anti-VEGF drugs in patients resistant to treatment with RBZ is important to achieve rapid resolution of fluids, especially intraretinal fluid. This would improve the visual outcomes before subsequent deleterious effects of chronicity start to play a major role on results. It could also diminish the burden and risks associated with more frequent intravitreal injections. The main limiting factors of this study were the retrospective design and lack of control group.

\section{Abreviations}

VA: Visual acuity; CMT: Central macular thickness; AFL: Aflibercept; RBZ: Ranibizumab; nAMD: Neovascular age-related macular degeneration; OCT: Optical coherence tomography; VEGF: Vascular endothelial growth factor; PRN: Pro re nata.

\section{Acknowledgements}

Not applicable.

\section{Authors' contributions}

LSQM interpreted the patient data and was a major contributor in writing the manuscript. MFSU collected data, performed the statistical analysis and was a major contributor in writing the manuscript. BVV collected data. SK and CVSR contributed to the interpretation of patient data. All authors read and approved the final manuscript.

\section{Funding}

Not applicable.

\section{Availability of data and materials}

The data that support the findings of this study are available from Hospital Oftalmológico de Brasilia but restrictions apply to the availability of these data, which were used under license for the current study, and so are not publicly available. Data are however available from the authors upon reasonable request and with permission of Hospital Oftalmológico de Brasilia.

\section{Ethics approval and consent to participate}

Project reviewed and approved by the Research Ethics Committee of Hospital Oftalmológico de Brasília. CAAE project number: 91652418.2.0000.5667.

\section{Consent for publication}

Not applicable.

\section{Competing interests}

The authors declare that they have no competing interests.

\section{Author details}

1 Department of Retina and Vitreous of Hospital Oftalmológico de Brasília, Brasília, Brazil. ${ }^{2}$ Hospital Oftalmológico de Brasília, Brasília, Brazil. ${ }^{3}$ Federal University of São Paulo (UNIFESP), São Paulo, Brazil.

Received: 14 October 2019 Accepted: 2 March 2020

Published online: 14 May 2020

\section{References}

1. Gass MD, Donald J. Sterioscopic atlas of macular diseases: diagnosis and treatment. Washington, DC: The C. V Mosby Company; 1987. p. 60-97.

2. Ager MD, Rama D, Meiler MD, et al. Age-related macular degeneration. N Engl J Med. 2008;358:606-17.

3. Stewart MW, Rodenfeld PJ. Predicted biological activity of intravitreal VEGF Trap. Br J Opthalmology. 2008;92(5):667-8.

4. Yang J, Wang $X$, Fuh $G$, et al. Comparison of binding characteristics and in vitro activities of three inhibitors of vascular endotelial growth factor $A$. Mol Pharm. 2014:11(10):3421-30.

5. Papadopoulos N, Martin J, Ruan Q, et al. Binding and neutralization of vascular endotelial growth fator (VEGF) and related ligands by VEGF Trap, rabizumanb and bevacizumab. Angiogenesis. 2012;15(2):171-85.

6. Rosenfeld PJ, Brown DM, Heier JS, Boyer DS, Kaiser PK, Chung CY, Kim RY, MARINA Study Group. Ranibizumab for neovascular age-related macular degeneration. N Engl J Med. 2006;355(14):1419-31.

7. Brown DM, Michels M, Kaiser PK, Heier JS, Sy JP, lanchulev T, NCHOR Study group. Ranibizumab versus verteporfin photodynamic therapy for neovascular age-related macular degeneration: Two-year results of the ANCHOR study. Ophthalmology. 2009;116(1):57-65.

8. Kumar N, Marsiglia M, Mrejen S, Fung AT, Slakter J, Sorenson J, et al. Visual and anatomical outcomes of intravitreal aflibercept in eyes with persistent subfoveal fluid despite previous treatments with ranibizumab in patients with neovascular age-related macular degeneration. Retina. 2013;33(8):1605-12.

9. CATT Research Group, Martin DF, Maguire MG, Ying GS, Grunwald JE, Fine SL, et al. Ranibizumab and bevacizumab for neovascular age-related macular degeneration. N Engl J Med. 2011:364(20):1897-908.

10. Comparison of Age-related Macular Degeneration Treatments Trials (CATT) Research G, Martin DF, Maguire MG, Fine SL, Ying GS, Jaffe GJ, Grunwald JE, Toth C, et al. Ranibizumab and bevacizumab for treatment of neovascular age-related macular degeneration: two-year results. Ophthalmology. 2012;119(7):1388-98.

11. Binder S. Loss of reactivity in intravitreal anti-VEGF therapy: tachyphylaxis or tolerance? Br J Ophthalmol. 2012;96(1):1-2.

12. Busbee BG, Ho AC, Brown DM, Heier JS, Suner IJ, Li Z, Rubio RG, Lai P, HARBOR Study Group. Twelve-month efficacy and safety of $0.5 \mathrm{mg}$ or $2.0 \mathrm{mg}$ ranibizumab in patients with subfoveal neovascular age-related macular degeneration. Ophthalmology. 2013;120(5):1046-56.

13. Wong TP. Two Year SAVE Outcomes: $2.0 \mathrm{mg}$ ranibizumab for recalcitrant neovascular AMD. Ophthalmology. 2013:120(9):1945-6.

14. Schmidt-Erfurth U, Kaiser PK, Korobelnik JF, et al. Intravitreal aflibercept injection for neovascular age-related macular degeneration ninety-sixweek results of the VIEW studies. Ophthalmology. 2014;121(1):193-201.

15. Egho MS, Sorensen TL. Tachyphylaxis during treatment of exudative age-related macular degeneration with ranibizumab. Br J Ophthalmol. 2012;96(1):21-3. 
16. Gokce G, Durukan AH, Koylu MT, Kucukevcilioglu M. Efficacy of aflibercept on exudative age-related macular degeneration in patients exhibiting complete ranibizumab resistance and tachyphylaxis. Arq Bras Oftalmol. 2016;79(6):384-9.

17. Gasperini JL, Fawzi AA, Khondkaryan A, Lam L, Chong LP, Eliott D, et al. Bevacizumab and ranibizumab tachyphylaxis in the treatment of choroidal neovascularisation. Br J Ophthalmol. 2012;96(1):14-20.

18. Li Jianqing, Jiayi Xu, Chen Yiyi, Zhang Jiaju, Cao Yihong, Peirong Lu. Efficacy comparison of intravitreal anti-VEGF therapy for three subtypes of neovascular age-related macular degeneration: a systematic review and meta-analysis. J Ophthalmol. 2018;2018:1425707. https://doi. org/10.1155/2018/1425707

19. Ashraf M, Souka A, Adelman RA. Age-related macular degeneration: using morphological predictors to modify current treatment protocols. Acta Ophthalmol. 2018;96(2):120-33.

20. Finger RP, Wickremasinghe SS, Baird PN, Guymer RH. Predictors of antiVEGF treatment response in neovascular age-related macular degeneration. Surv Ophthalmol. 2014;59(1):1-18.

21. Avgikos KN, Horgan SE, Sivaraj RR, Hu K. Tachyphylaxis and bevacizumab. Ophthalmology. 2009;116(9):1831-2.

22. Schaal S, Kaplan HJ, Tezel TH. Is there tachyphylaxis to intravitreal antivascular endothelial growth factor pharmacotherapy in age-related macular degeneration? Ophthalmology. 2008;115(12):2199-205.

23. Almony A, Mansouri A, Shah GK, Blinder KJ. Efficacy of intravitreal bevacizumab after unresponsive treatment with intravitreal ranibizumab. Can J Ophthalmol. 2011;46(2):182-5.

24. Jaffe GJ, Martin DF, Toth CA, et al. Macular morphology and visual acuity in the comparison of age-related macular degeneration treatments trials. Ophthalmology. 2013;120:1860-70.

25. Gianniou C, Dirani A, Jang L, Mantel I. Refractory intraretinal or subretinal fluid in neovascular age-related macular degeneration treated with intravitreal ranizubimab: functional and structural outcome. Retina. 2015;35:1195-201.

26. Wickremasinghe SS, Janakan V, Sandhu SS, Amirul-Islam FM, Abedi F, Guymer RH. Implication of recurrent or retained fluid on optical coherence tomography for visual acuity during active treatment of neovascular age-related macular degeneration with a treat and extend protocol. Retina. 2016;36:1331-9.

27. Waldstein SM, Philip AM, Leitner R, et al. Correlation of 3-dimensionally quantified intraretinal and subretinal fluid with visual acuity in neovascular age-related macular degeneration. JAMA Ophthalmol. 2016;134:182-90.

28. Jang L, Gianniou C, Ambresin A, Mantel I. Refractory subretinal fluid in patients with neovascular age-related macular degeneration treated with intravitreal ranibizumab: visual acuity outcome. Graefes Arch Clin Exp Ophthalmol. 2015;253:1211-6.

29. Waldstein SM, Wright J, Warburton J, Margaron P, Simader C, SchmidtErfurth U. Predictive value of retinal morphology for visual acuity outcomes of different ranibizumab treatment regimens for neovascular AMD. Ophthalmology. 2016;123:60-9.

30. Sato T, Suzuki M, Ooto S, Spaide RF. Multimodal imaging findings and multimodal vision testing in neovascular age-related macular degeneration. Retina. 2015;35:1292-302.

31. Grunwald JE, Daniel E, Huang J, et al. Risk of geographic atrophy in the comparison of age-related macular degeneration treatments trials. Ophthalmology. 2014;121:150-61.

\section{Publisher's Note}

Springer Nature remains neutral with regard to jurisdictional claims in published maps and institutional affiliations.
Ready to submit your research? Choose BMC and benefit from:

- fast, convenient online submission

- thorough peer review by experienced researchers in your field

- rapid publication on acceptance

- support for research data, including large and complex data types

- gold Open Access which fosters wider collaboration and increased citations

- maximum visibility for your research: over 100M website views per year

At BMC, research is always in progress.

Learn more biomedcentral.com/submissions 